\title{
Bureau d'expertises extrajudiciaires de la FMH - Rapport annuel 2009
}

\section{Lucia Rabia ${ }^{a}$,}

Nathalie Favre ${ }^{b}$

a avocate, service juridique de la FMH

b lic. en droit, service juridique de la FMH
En 2009, le Bureau d'expertises extrajudiciaires de la FMH a procédé à l'établissement de 68 expertises. Les experts ont conclu à une faute de traitement dans 34 cas et n'ont constaté aucune faute dans 33 autres cas. Dans un cas, il n'a pas été possible de répondre à la question de la faute.

Le Bureau d'expertises de la FMH n'est pas compétent pour tous les litiges. Sa tâche est de mandater une expertise lorsque le patient, soigné en Suisse, présume que le médecin exerçant en pratique privée ou à l'hôpital a commis une faute de diagnostic et/ou de traitement qui a conduit à une atteinte considérable à sa santé et lorsque, de surcroît, le patient n'a pu trouver d'accord sans expertise avec l'assureur responsabilité civile du médecin ou de l'hôpital. Une autre condition est qu'aucun tribunal n'a été saisi du litige ni n'a prononcé de jugement à ce sujet. Depuis l'entrée en vigueur du nouveau règlement en 2002, les informations détaillées sur l'histoire du cas que nous recevons du patient, du médecin/de son assureur ou de l'hôpital permettent de mieux saisir les problèmes à examiner. Le Bureau d'expertises extrajudiciaires de la FMH peut ainsi octroyer plus sûrement son mandat d'expertise à l'équipe d'experts adéquate pour le cas en présence. En outre, la complexité de nombreux cas est d'emblée reconnaissable: dans bien des situations, l'équipe d'experts a dû être composée de représentants de deux ou trois disciplines médicales différentes.

\section{Statistiques du Bureau d'expertises pour I'année 2009}

\section{Méthode}

Durant l'exercice écoulé, 68 expertises ont été menées à bien alors que leur nombre s'était élevé à 69 durant l'exercice précédent. Dans un bon tiers des cas examinés, il s'est agi d'analyser exclusivement des traitements prodigués par des médecins en cabinet privé. Quant au reste des cas (pratiquement les deux tiers), ils ont porté soit exclusivement sur l'analyse de traitements hospitaliers, soit sur des traitements impliquant conjointement des cabinets privés et des hôpitaux. Les expertises réalisées en 2009 ont nécessité l'intervention de 17 équipes multidisciplinaires d'experts.

En cas d'expertise multidisciplinaire, la classification s'effectue selon la discipline la plus touchée par le cas. Exemple: s'il est fait appel à une équipe d'experts principalement en gynécologie et secondairement en anesthésiologie et qu'une faute est reconnue en gynécologie, et non pas en anesthésiologie, l'expertise sera classée dans la catégorie «gynécologie, faute constatée». $\mathrm{Si}$, dans le même cas, une faute est reconnue en anesthésiologie, et non pas en gynécologie, l'expertise sera classée exclusivement dans la catégorie «anesthésiologie, faute constatée». Si une faute est reconnue dans ces deux disciplines, l'expertise apparaît dans la statistique sous "gynécologie, faute constatée».

La statistique reflète ainsi le résultat déterminant pour le patient et non pas la mesure du travail total fourni par les experts.

\section{Causalité entre la faute et le dommage à la santé} La réponse à la question de savoir si une faute a été commise dans le diagnostic ou le traitement ne clôt pas l'expertise dans chaque cas. Si des fautes ont effectivement été constatées, il convient alors de déterminer si elles sont aussi à l'origine du dommage à la santé évoqué par le patient. Le droit de ce dernier à une réparation n'existe qu'à partir du moment où la causalité entre la faute et le dommage est reconnue.

Les cas dans lesquels les experts constatent certes une faute, mais pas de rapport de causalité entre celle$\mathrm{ci}$ et le dommage, sont relativement nombreux. $\mathrm{Ou}$, formulé de manière positive: en médecine comme ailleurs, les fautes n'ont pas toutes, heureusement, des conséquences négatives ou graves. L'expert doit donc s'exprimer sur l'état de santé qui serait celui du patient si la faute en question ne s'était pas produite.

La statistique établie depuis des années ne recense pas ce critère de manière explicite. Pour 2009, la causalité entre la faute constatée et le dommage a été clairement reconnue ou reconnue comme étant très probable dans un peu plus de la moitié des dossiers concluant à l'existence d'une faute. Toutefois, dans une petite moitié des cas avec fautes avérées, la causalité a été rejetée ou le lien entre faute et dommage a été considéré uniquement comme possible. Il est souvent difficile de quantifier l'influence d'un seul facteur, par exemple d'une faute de traitement, sur le résultat global insatisfaisant. Souvent, d'autres facteurs déterminants influencent le résultat, comme un pronostic de guérison préalablement défavorable dans tel cas particulier, ou des maladies supplémentaires.

\section{Information médicale au patient et communication entre médecin et patient}

L'information médicale au patient à elle seule ne peut pas faire l'objet d'une expertise de la FMH. Elle peut toutefois être abordée parallèlement à la faute de diagnostic et/ou de traitement supposée. De manière tout 
à fait générale, nous aimerions par expérience souligner combien il est important que cette information du patient soit suffisamment documentée.

Dans plusieurs cas, les experts sont en effet parvenus à la conclusion qu'il n'y avait pas de faute de diagnostic ni de traitement, mais que l'information aux patients sur le plan médical était insuffisante ou faisait défaut ou qu'elle était lacunaire, voire pas du tout documentée.

A diverses reprises, il s'est aussi avéré que la communication entre médecins et patients laissait à désirer. Si les résultats du traitement ne correspondent

Tableau 1

Vue d'ensemble globale 1982-2009.

\begin{tabular}{|c|c|c|c|c|}
\hline & $\begin{array}{l}\text { Expertises } \\
\text { établies }\end{array}$ & $\begin{array}{l}\text { Fautes de diagnostic et } \\
\text { de traitement avérées }\end{array}$ & $\begin{array}{l}\text { Fautes de diagnostic et } \\
\text { de traitement niées }\end{array}$ & $\begin{array}{l}\text { Fautes de diagnostic et } \\
\text { de traitement indéterminées }\end{array}$ \\
\hline Toute la Suisse 1982-2008 & 3165 & 1031 & 2042 & 92 \\
\hline Suisse alémanique et Tessin 2009 & 31 & 17 & 14 & 0 \\
\hline Suisse romande 2009 & 37 & 17 & 19 & 1 \\
\hline Toute la Suisse 2009 & $68(100 \%)$ & $34(50,0 \%)$ & $33(48,5 \%)$ & $1(1,5 \%)$ \\
\hline Total 1982-2009 & $3233(100 \%)$ & $1065(32,9 \%)$ & $2075(64,2 \%)$ & $93(2,9 \%)$ \\
\hline Total des 10 dernières années 2000-2009 & $904(100 \%)$ & $398(44,0 \%)$ & 491 (54,3\%) & $15(1,7 \%)$ \\
\hline
\end{tabular}

Tableau 2

Résultats par spécialité 1982-2009.

\begin{tabular}{|c|c|c|c|c|}
\hline & $\begin{array}{l}\text { Expertises } \\
\text { établies }\end{array}$ & $\begin{array}{l}\text { Fautes de diagnostic et } \\
\text { de traitement avérées }\end{array}$ & $\begin{array}{l}\text { Fautes de diagnostic et } \\
\text { de traitement niées }\end{array}$ & $\begin{array}{l}\text { Fautes de diagnostic et } \\
\text { de traitement indéterminées }\end{array}$ \\
\hline Médecine générale & 228 & 81 & 137 & 10 \\
\hline Anesthésiologie & 116 & 37 & 76 & 3 \\
\hline Chirurgie & 804 & 278 & 499 & 27 \\
\hline Dermatologie & 29 & 9 & 18 & 2 \\
\hline Gastro-entérologie & 14 & 2 & 12 & 0 \\
\hline Gynécologie et obstétrique & 395 & 151 & 238 & 6 \\
\hline Chirurgie de la main & 48 & 17 & 30 & 1 \\
\hline Chirurgie cardiaque et vasculaire thoracique & 25 & 8 & 16 & 1 \\
\hline Médecine interne & 211 & 63 & 144 & 4 \\
\hline Cardiologie & 19 & 11 & 8 & 0 \\
\hline Chirurgie maxillo-faciale & 22 & 3 & 19 & 0 \\
\hline Chirurgie pédiatrique & 14 & 4 & 10 & 0 \\
\hline Psychiatrie pédiatrique & 1 & 0 & 1 & 0 \\
\hline Néphrologie & 2 & 0 & 2 & 0 \\
\hline Neurochirurgie & 82 & 22 & 58 & 2 \\
\hline Neurologie & 24 & 7 & 16 & 1 \\
\hline Oncologie & 6 & 3 & 3 & 0 \\
\hline Ophtalmologie & 121 & 35 & 81 & 5 \\
\hline Chirurgie orthopédique & 596 & 211 & 370 & 15 \\
\hline Oto-rhino-laryngologie ORL & 113 & 24 & 85 & 4 \\
\hline Pédiatrie & 63 & 26 & 34 & 3 \\
\hline Pathologie & 6 & 4 & 2 & 0 \\
\hline Pharmacologie & 2 & 2 & 0 & 0 \\
\hline Médecine physique et réadaptation & 13 & 3 & 9 & 1 \\
\hline Chirurgie plastique, reconstructive et esthétique & 124 & 27 & 95 & 2 \\
\hline Pneumologie & 1 & 1 & 0 & 0 \\
\hline Psychiatrie & 15 & 7 & 8 & 0 \\
\hline Radiologie & 48 & 11 & 34 & 3 \\
\hline Radio-oncologie & 1 & 1 & 0 & 0 \\
\hline Rhumatologie & 16 & 5 & 11 & 0 \\
\hline Urologie & 74 & 12 & 59 & 3 \\
\hline Total 1982-2009 & 3233 & 1065 & 2075 & 93 \\
\hline
\end{tabular}


pas entièrement aux attentes du patient ou si le traitement prend subitement une mauvaise tournure, une communication insuffisante de la part des médecins risque d'éveiller ou de renforcer chez les patients la présomption qu'une faute a été commise.

\section{Limite de la valeur probante de la statistique}

Le nombre peu élevé de 68 expertises terminées en 2009 incite en lui-même à la prudence si l'on veut en tirer des conclusions éventuelles. Cette statistique est peu représentative de la situation en matière de responsabilité civile dans les hôpitaux et chez les médecins en Suisse. Un grand hôpital cantonal non universitaire est confronté, à lui seul, entre vingt et trente cas de responsabilité civile chaque année.

Cette statistique montre donc uniquement combien d'expertises ont été établies dans les diverses disciplines par le Bureau d'expertises de la FMH et dans combien d'entre elles une faute de diagnostic et/ou de traitement a été constatée ou niée. Le petit nombre de données à disposition et le manque de valeurs comparatives ne permettent pas d'en tirer d'autres conclusions. On ne saurait donc, par exemple, procéder sur cette base à des calculs pour établir le pourcentage de fautes par discipline médicale ou dans la médecine en général.

Le pourcentage de fautes reconnues a de nouveau changé par rapport à l'exercice précédent et s'élève à 50\% pour l'année 2009 (contre $46 \%$ en 2008 et près de $35 \%$ en 2007). Ici également, la prudence est de mise si l'on veut procéder à une interprétation de cette modification. Il suffit qu'un petit nombre de cas soit bouclé durant l'année précédente, en cours ou suivante et apparaisse dans la statistique pour modifier sensiblement ce pourcentage. Le Bureau d'expertises extrajudiciaires est tenu de procéder avec fairplay et veille à ce que chaque dossier soit traité correctement.

\section{Entretien préliminaire par téléphone, adresses, documents}

Depuis des années, le Bureau d'expertises extrajudiciaires de la FMH donne l'occasion aux patients, à leurs avocats et à d'autres conseillers de discuter du cas d'espèce, par téléphone avec le Bureau d'expertises, avant le dépôt définitif de la demande. Sur la base de ces recherches préliminaires, où une faute peut-elle avoir été commise et qui en serait responsable? Quelles sont les autres causes de fautes possibles? En quoi pourrait consister le dommage à la santé? Quels sont les éléments particuliers que doit indiquer le Bureau d'expertises aux délégués des sociétés de discipline médicale qui proposent des experts? etc. Ces discussions préalables nécessitent peut-être une demi-heure ou une heure, mais elles permettent d'éviter nombre de questions ultérieures et font gagner un temps précieux dans l'intérêt de la procédure d'expertise.

Les documents nécessaires au dépôt d'une demande d'expertise peuvent être obtenus auprès du Bureau d'expertises extrajudiciaires de la $\mathrm{FMH}$, Case postale 6159, 3001 Berne, Tél. 03138058 10, Fax: 0313805819.

D'autres informations peuvent être obtenues sous www.fmh.ch $\rightarrow$ Service $\rightarrow$ Bureau d'expertises extra-judiciaires.
Ce qui n'apparaît pas dans la statistique, c'est toujours le grand investissement de temps et de ressources engagé dans des demandes qui ne conduiront finalement pas à une expertise. Patients, avocats, médecins, assurances et autres institutions s'adressent, avec des questions multiples et variées, au Bureau d'expertises extrajudiciaires qui tente, dans la mesure du possible, de leur donner des informations utiles, même si les problèmes exposés n'entrent pas dans son domaine de compétences. Il est aussi souvent difficile de faire comprendre à un patient que le Bureau d'expertises de la FMH n'offre pas une procédure informelle et unilatérale et que les complications survenues ou les attentes de guérison déçues ne peuvent pas toutes conduire à une expertise.

\section{Assurance-qualité}

L'assurance-qualité revêt une grande importance dans la procédure suivie par le Bureau d'expertises. Les démarches suivantes y contribuent:

- Les sociétés de discipline médicale proposent, pour chaque cas particulier, des experts qui acceptent le mandat confié avec l'accord de l'ensemble des parties concernées. Dès le début, une équipe est constituée avec des experts de différentes disciplines lorsque le cas l'exige. Le but visé est d'assurer une évaluation par des personnes compétentes en la matière, de manière analogue à un traitement médical.

- En outre, le schéma destiné aux experts et utilisé depuis des années s'avère très utile car il les aide dans leur tâche d'élaborer une expertise qui permette aux parties de régler le litige.

- Un instrument particulier de l'assurance-qualité est la relecture du projet d'expertise par le service juridique de la FMH. A quelques exceptions près, les patients approuvent cette manière de procéder. La tâche des deux juristes est ainsi de soutenir les experts dans la rédaction d'une expertise qui soit complète, pertinente et, surtout, compréhensible pour des profanes.

\section{Formation des experts}

Les juristes du Service juridique de la FMH ont participé à diverses rencontres dont le but est de former les médecins à l'expertise médicale ou qui abordent la question de la responsabilité civile du médecin. Pendant l'exercice sous revue, elles sont intervenues lors des formations en expertises médicales de Swiss Insurance Medicine (SIM), dans le cadre des séminaires pour experts proposés par la Société suisse de gynécologie et obstétrique (SSGO), ainsi qu'à l'occasion du congrès scientifique du Collège International de Chirurgiens (CIC).

\section{Durée de la procédure}

La durée de la procédure est régulièrement critiquée, avant tout par les patients qui attendent avec im- 
patience une réponse à leurs questions. Il arrive rarement qu'une expertise puisse être close moins d'un an après l'envoi de la demande. D'autre part, une procédure réglementée, transparente et acceptable par tous prend du temps. Selon les cas, la recherche d'experts compétents dure à elle seule très longtemps.

Lorsque plusieurs experts ont été mandatés, chaque étape requiert plus de temps, depuis l'audition et l'examen du patient jusqu'à la rédaction finale de l'expertise. Il faut souligner que la charge de travail de nombreux experts est telle qu'ils trouvent à peine le temps nécessaire pour effectuer ce genre de mandats supplémentaires dans le délai souhaité; la plupart y sacrifient même une partie de leurs moments de loisir.

\section{Conseil scientifique}

Le Conseil scientifique surveille l'activité du Bureau d'expertises extrajudiciaires sur mandat du Comité central de la FMH. Il n'a pas de compétence décisionnelle en ce qui concerne les différents cas traités mais décharge le Comité central de son activité de surveillance. Au cours de l'exercice sous revue, le Conseil scientifique a tenu deux séances et a examiné par sondage quelques dossiers d'expertise.

Le Conseil scientifique se compose des membres suivants: le Dr Bruno Lerf, président, le Dr Thomas Froesch et Me Massimo Pergolis, avocat.

\section{Personnel}

Madame Susanne Friedli est responsable du Bureau d'expertises extra-judiciaires qui a été réorganisé en été 2008. Monsieur Sébastian Lerch, de langue maternelle française, est son suppléant depuis janvier 2009. Il traite les dossiers de la Suisse romande. Le Bureau d'expertises extra-judiciaires est supervisé par Madame Lucia Rabia, avocate, et Madame Nathalie Favre, licenciée en droit, toutes deux en fonction au Service juridique de la FMH.

\section{Remerciements}

Pour pouvoir fonctionner, le Bureau d'expertises extrajudiciaires a besoin de la collaboration de nombreuses personnes. Nous remercions les sociétés de discipline médicale et leurs délégués de leur précieux soutien ainsi que les experts pour la grande somme de travail accomplie en vue d'éclaircir les cas. Nous remercions les médecins traitants ainsi que les directions d'hôpitaux qui ont coopéré de manière ouverte et correcte à la réalisation d'une expertise demandée par un patient.

Mme Susanne Friedli et Mr Sébastian Lerch sont chargés du traitement des dossiers, depuis la réception de la première demande jusqu'à l'envoi des rapports d'expertise. Ils sont les interlocuteurs de toutes les parties et fournissent un grand travail de coordination et de conseils. Nous leur adressons ici nos vifs remerciements pour leur engagement.

\section{Le marché de l'emploi dans le Bulletin des médecins suisses: les postes de médecins en Suisse - mise à jour hebdomadaire.}

Bulletin des médecins suisses Bollettino dei medici svizzeri Schweizerische Ärztezeitung

C'est ici que vous trouverez le poste que vous cherchez ou votre candidat idéal.

Le Bulletin des médecins suisses, organe officiel de la FMH et de FMH-Services, est un hebdomadaire publiant 45 numéros par an. Il constitue le marché central des offres d'emploi pour les médecins en Suisse.

Toutes les annonces sont publiées immédiatement aussi bien dans la version imprimée que dans la version en ligne sous www.bullmed.ch (en français) et www.saez.ch (en allemand).

Le Bulletin des médecins suisses, revue de pointe des médecins en Suisse, est une publication des Editions médicales suisses $\mathrm{EMH}$.

Nous vous souhaitons le succès!

Contact:

EMH Schweizerischer Ärzteverlag AG | Farnsburgerstrasse 8 | CH-4132 Muttenz

Tél. 0614678554 | Fax 0614678556 | E-Mail: stellenmarkt@emh.ch | Internet: www.emh.ch
EMH Schweizerischer Ärzteverlag AG Editores Medicorum Helveticorum 